\begin{tabular}{|c|c|c|c|c|c|}
\hline MUNIBE Antropologia-Arkeologia & $n^{\circ} 72$ & $111-122$ & DONOSTIA & 2021 & ISSN 1132-2217 • eISSN 2172-4555 \\
\hline
\end{tabular}

\title{
Simbolismo de la Edad del Bronce en la Meseta oriental: un depósito cerámico con Arte Pospaleolítico en la cueva del Cojillo (Checa, Guadalajara)
}

\author{
Bronze Age symbolism in the eastern Meseta: a pottery deposit \\ with schematic art at El Cojillo cave (Checa, Guadalajara)
}

PALABRAS CLAVES: Bronce final, Grabado Pospaleolítico, C14, Cerámica incisa.

GAKO-HITZAK: Amaierako Brontze garaia, Paleolito osteko grabatua, C14, moztutako zeramika

KEY WORDS: Late Bronze, Engravings, Schematic Art, Radiocarbon date, Incised ceramic.

Javier ARAGONCILLO(1), Ignacio TRIGUERO(2)

\section{RESUMEN}

Se presentan los primeros trabajos arqueológicos efectuados en la cueva del Cojillo (Checa, Guadalajara). Durante la intervención se extrajo un depósito cerámico in situ cuyo contenido ha aportado una fecha C14 que permite situarlo en el Bronce final. Asimismo, la cavidad contiene grafías que, por su carácter técnico y temático, se aproximan al Arte Pospaleolítico en cueva de la Submeseta norte. Las estribaciones occidentales del sistema Ibérico sufren, durante el Bronce final, profundos cambios culturales que sitúan este yacimiento en un momento clave. Una de sus consecuencias es el abandono de cavidades para usos habitacionales o simbólicos. La cueva del Cojillo apunta hacia una perduración de prácticas enraizadas en el Bronce durante el final del II milenio a.C. Se ofrece un registro pormenorizado del depósito y los paneles, una reevaluación de la extensión de este horizonte gráfico y una aproximación a la significación del conjunto.

\section{LABURPENA}

Cojilloko (Checa, Guadalajara) kobazuloan egindako lehen lan arkeologikoak aurkezten ditugu. Esku-hartzean, zeramikazko multzoa atera zuten in situ eta bertako edukiari esker C14 data zehaztu denez, amaierako Brontze garaikoa dela esan dezakegu. Era berean, kobazuloan dauden grafien izaera teknikoa eta tematikoa dela eta, iparraldeko azpimesetako kobazuloan Paleolito ondorengo artetik gertu daudela esan dezakegu. Iberiar Sistemako mendebalde inguruko mendietan, amaierako Brontzean, aldaketa kultural handiak gertatu ziren eta aztarnategi hau, hain zuzen ere, une erabakigarri hartan kokatu behar dugu. Aldaketa haien ondorioetako bat izan zen kobazuloak bizitegi gisa erabiltzeari edo erabilera sinbolikoetarako bideratzeari utzi ziotela. Cojilloko kobazuloan, Brontze garaian errotutako ohiturek K.a II. milurtekoaren amaieran luzaroan iraun zutela dirudi. Zeramiken eta panelen erregistroa egin dugu, eta horizonte grafiko horren hedaduraren ebaluazio berri bat ikus dezakegu, baita multzoaren esanahiarekiko hurbilketa bat ere.

\section{ABSTRACT}

The archaeological study carried out in the Cojillo cave (Checa, Guadalajara) is presented. During the intervention, an in situ ceramic deposit was extracted, and its content gave a late Bronze age radiocarbon date. Likewise, the cavity contains rock art that, due to technical and thematic features, can be included in the Duero valley post-Palaeolithic cave art group. It is the first example of its type in Southern Iberian Inland. The western foothills of the Iberian system undergo, during the final Bronze, profound cultural changes that place this site at a key moment. One of its consequences is the abandonment of cavities for habitat or symbolic uses. The Cojillo cave points to a persistence of practices rooted in the Bronze during the end of the second millennium BC. A detailed record of the deposit and the panels is offered, a reassessment of the extension of this graphic expression is proposed and an approximation to the significance of the whole is discussed.

\section{INTRODUCCIÓN}

Durante las prospecciones realizadas a lo largo de 2017 a raíz del proyecto "El Paleolítico en el Alto Tajo" dirigido por el Dr. J. M. Maílo y el primer firmante del artículo en varios términos municipales de la comarca de Molina de Aragón-Alto Tajo, se examinaron varias cavidades en el valle del río Hoz Seca, en el término municipal de Checa, Guadalajara. En la cueva del Cojillo se detectaron numerosos galbos atípicos de cerámica facturada a mano, un fragmento de posible molino barquiforme en arenisca, así como un conjunto vascu-

\footnotetext{
(1) jarrio70@gmail.com. Centro de Estudios de Molina y Alto Tajo. C/Cuartell ,3 1D, 19300 Molina de Aragón, Guadalajara. ORCID: 0000-00023684-7217.

(2) Área de Prehistoria, Universidad de Alcalá. Calle Colegios, 2, 28801 Alcalá de Henares, Madrid. Ignaciotriguero@gmail.com. ORCID: 00000003-4685-0134.
} 
lar y varios paneles con grafías. También aparecieron materiales cerámicos elaborados a mano igualmente atípicos en la entrada de la cercana cueva del Tornero, con un dispositivo gráfico actualmente en estudio.

La ocupación de cavidades y abrigos a lo largo del curso alto del Tajo y sus afluentes es habitual a lo largo de la Prehistoria reciente, como constatamos en este trabajo y durante la realización de las Cartas Arqueológicas (2007-2009) para la Junta de Comunidades de Castilla-La Mancha. Destacan las cuevas del Estrecho del Hornillo, la Hoya del Castillo (Peiro y Martín, 1990), el Moreno y la Misa, en el Tajo; Cojillo, Tornero o las Duchas en el río Hoz Seca y distintas localizaciones en Ios ríos Gallo, Arandilla y Ablanquejo. Hasta la fecha se han excavado tres yacimientos de estas características pertenecientes a la Prehistoria reciente en el este de Guadalajara: la cueva de los Casares, con datos poco concluyentes acerca de su ocupación (Barandiarán, 1973; Alcaraz-Castaño, 2015), la Cueva de la Hoz, en la que se documenta su uso funerario durante el Calcolítico (Balbín, 2000) y el abrigo del Llano, también con ocupación calcolítica (Balbín et al., 1989).

Debido al prolongado uso de las cavidades, que se extiende prácticamente hasta la actualidad, la presencia de materiales en superficie in situ es muy poco frecuente. La cueva del Cojillo permite plantear posibles relaciones entre los materiales y las grafías, habida cuenta de que ambos se encuentran en la localización deliberadamente escogida en el pasado.

\section{LOCALIZACIÓN Y DESCRIPCIÓN DE LA CAVIDAD}

El río Hoz Seca es el primer afluente significativo del Tajo en su margen derecha, con un recorrido de apenas 25 kms Excava en su curso medio un cañón labrado en materiales jurásicos y cretácicos tableados en las estribaciones occidentales del sistema Ibérico, en un área con altitudes medias de 1.500 m s. n. m. La cueva del Tornero, de grandes dimensiones, ejemplifica la intensa actividad kárstica del valle, que recibe, entre otras aportaciones, aguas filtradas del sumidero del poljé del CubiIlo, próximo a la localidad de Checa. La meseta superior del margen derecho del Hoz Seca está ocupada actualmente por terrenos de labor en activo y otros abandonados, con bosques de pinus sylvestris y juniperus sabina, además de herbáceas y aromáticas.

La cueva del Cojillo se encuentra a unos 1400 m.s.n.m., en la margen derecha del Hoz Seca. Se abre en la parte superior de los cantiles que forman el cañón, en el paraje denominado El Molatón, un espolón enmar-

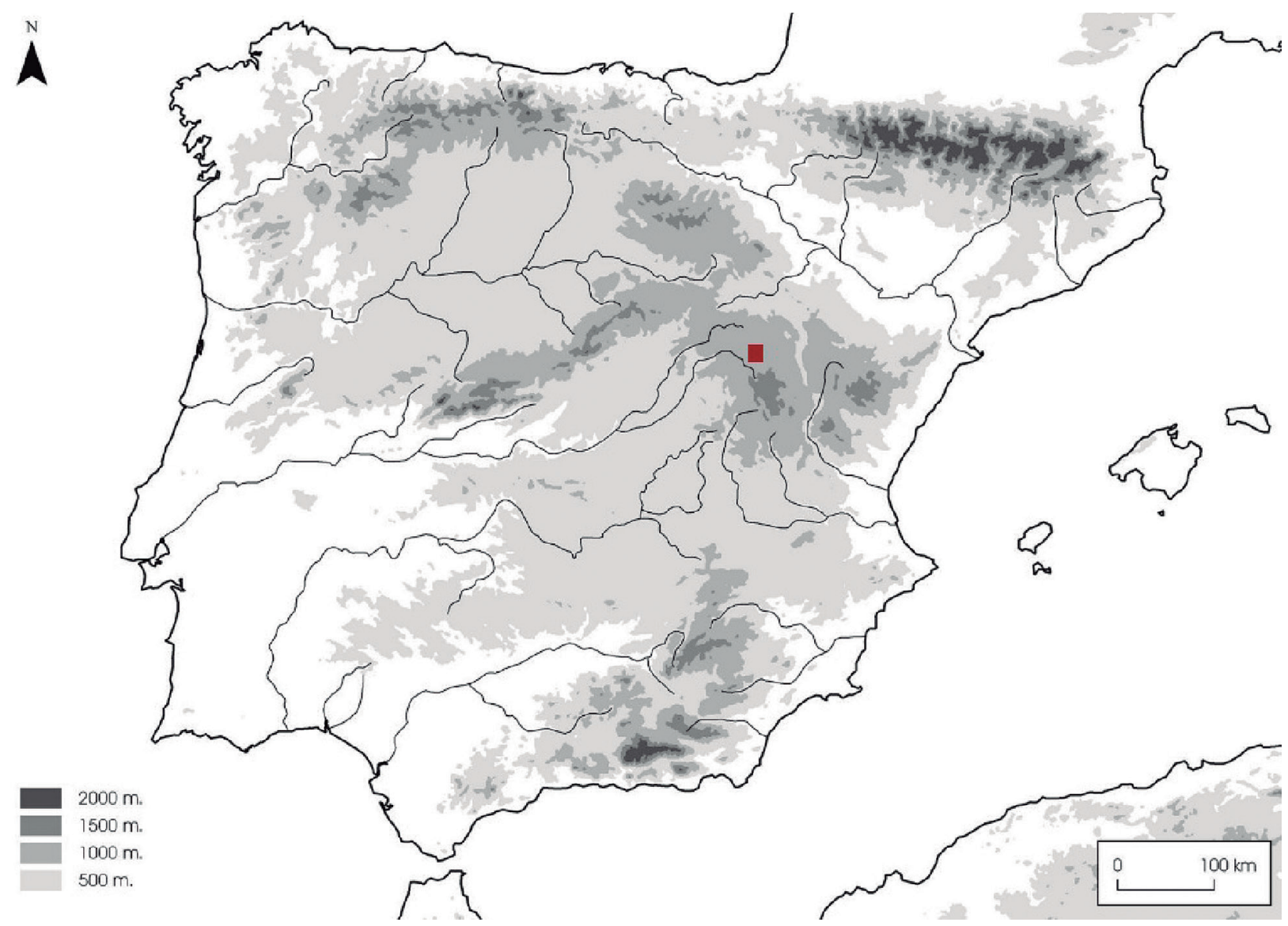

Fig. 1. Localización de la cueva del Cojillo. / Location of the Cojillo cave. 


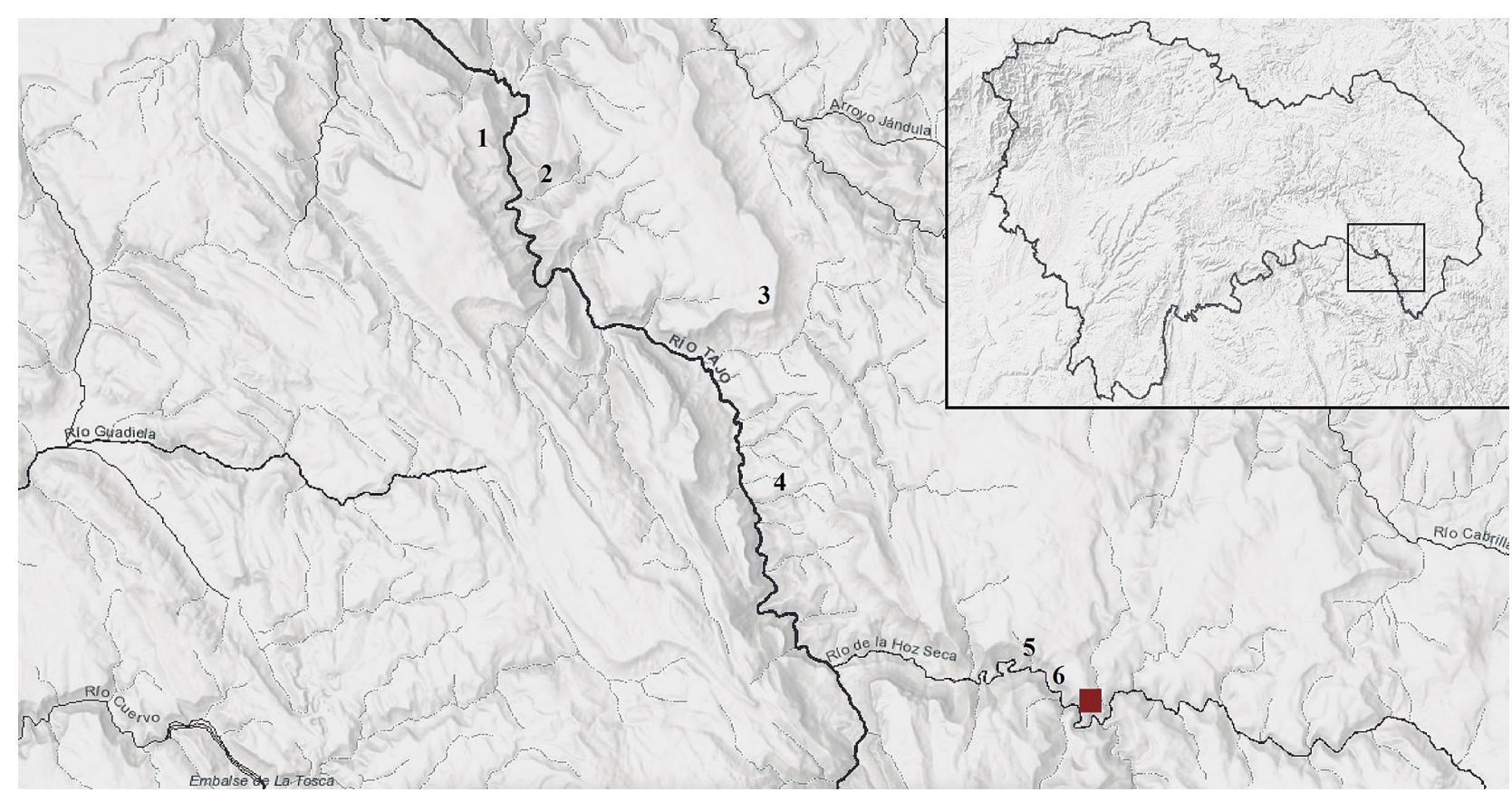

Fig. 2. Plano de situación de la cueva del Cojillo en relación con otras cuevas localizadas con restos de ocupación durante la Prehistoria reciente en el curso alto del Tajo y en el Hoz Seca. / Location map of the Cojillo cave in relation to other caves located with remains of occupation during recent Prehistory in the upper course of the Tagus river and the Hoz Seca river: 1 Estrecho del Hornillo, 2 Hoya del Castillo, 3 Cueva del Moreno, 4 Cueva de la Misa, 5 Cueva del Tornero, 6 Cueva de las Duchas.

cado por un pronunciado meandro que alberga en su cresta restos de dos asentamientos. El primero de ellos, muy alterado por la erosión, presenta restos de cerámica a mano y algún fragmento elaborado a torno, adscrito al Hierro. El segundo de ellos es medieval, posiblemente andalusí. Esta cavidad no aparece descrita en el Catálogo de Cavidades de Guadalajara (Fernández y Martín, 1990) por lo que se ha realizado una planimetría parcial.

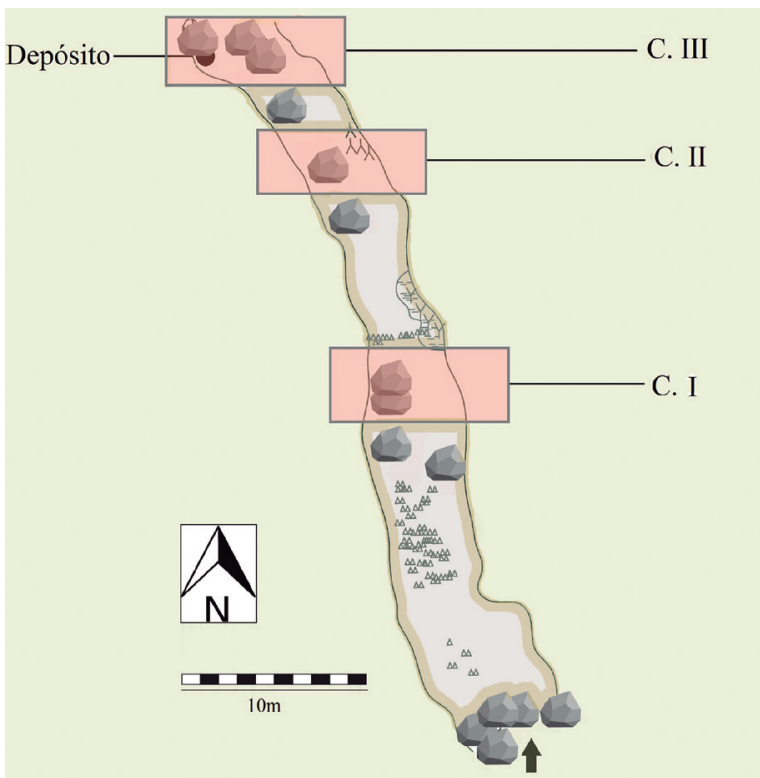

Fig. 3. Topografía parcial de la cueva del Cojillo. / Partial topography of the Cojillo cave.
La cavidad está orientada al sur y su boca aparece parcialmente cerrada por un muro trabado de mampostería en seco, parcialmente colmatado en el exterior. Da paso a un vestíbulo amplio y un corredor que discurre en dirección noroeste con medidas aproximadas de tres metros de alto y siete de ancho. Presenta en su primer tramo un suelo con aspecto de enlosado con clastos de mediano y gran tamaño. A lo largo de la entrada y del corredor inicial aparecen algunos fragmentos cerámicos a mano poco diagnósticos, entre los que destaca un fragmento de borde con decoración plástica en cordón digitado, en restos de depósito a un nivel superior del actual suelo. Este hecho apunta a un vaciado parcial de la cavidad para su uso como aprisco ganadero. A $25 \mathrm{~m}$ de la entrada aparecen posibles restos de un antiguo cierre, compuesto por una acumulación de piedras de pequeño y mediano tamaño, sin que se conserve ninguna hilada visible. Unos tres metros antes de llegar a este punto encontramos el primer sector decorado -Conjunto I-. A partir de aquí la cueva discurre por distintas salas divididas por obstáculos topográficos formados por bloques de gran tamaño. En la primera de ellas, en la parte izquierda del corredor se encuentra el Conjunto II, en el techo de la cavidad. Tras un estrecho paso se accede a una sala que gana en altura con el piso conformado por un caos de bloques de gran formato, en el que se localiza el Conjunto III, también cenital. Bajo este, a un metro de la pared de la sala, se localizó el depósito cerámico. Unos metros más al interior, un pequeño panel cierra el dispositivo gráfico. 


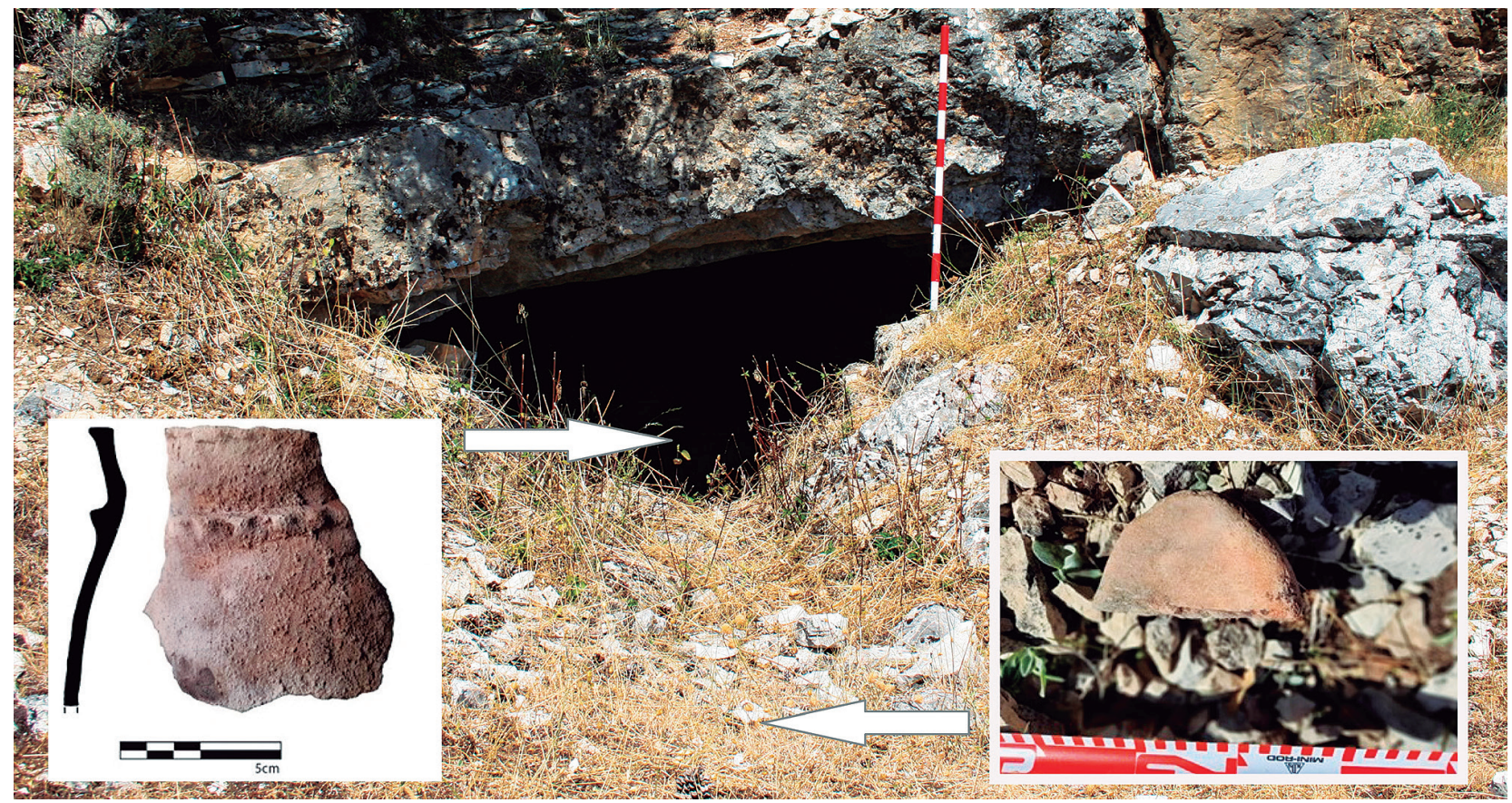

Fig. 4. Entrada de la cueva del Cojillo y materiales de las inmediaciones. Fragmento cerámico con borde digitado hallado en el corredor de entrada y fragmento de molino barquiforme, localizado en el exterior. / Cojillo cave entrance and materials from the surroundings. Ceramic fragment with fingered edge found in the entrance corridor and fragment of a barquiform mill, located outside the cave.

A partir de aquí la cavidad discurre $15 \mathrm{~m}$ en un pasillo estrecho entre bloques caídos, hasta alcanzar una amplia sala con grandes bloques de aspecto inestable en el techo y espeleotemas en las paredes laterales. La presencia de raíces indica la superficialidad de la cavidad en este punto. En esta sala no se detecta material arqueológico, restos de fauna ni grafías, aunque sí restos de madera quemada. Tras un estrecho paso detectamos los restos de una hemimandíbula inferior fósil de un carnívoro, en apariencia perteneciente a Ursus arctos, adherida al suelo del paso por la colada estalagmítica.

A partir de este punto la cavidad discurre entre arenas y arcillas de descalcificación y espeleotemas con restos de macrofauna fósil, así como una posible osera. Las paredes, blandas, conservan marcas de garras, posiblemente de oso, y aparecen en algún punto sobreexcavadas por la fauna que ocupó la cavidad. Distintas galerías cortan ortogonalmente la principal hasta su final. Destaca un punto de posible derrumbe, que pudiera indicar otra entrada sellada en el pasado. Estas galerías resultaron estériles en cuanto a restos arqueológicos, no así paleontológicos. Todo el desarrollo kárstico de la cavidad aparece poco activo, dominando los procesos por gravedad por infiltraciones superficiales de agua.

\section{EL DEPÓSITO}

\subsection{Descripción}

Se compone de dos recipientes cerámicos, ambos realizados a mano, dispuestos uno dentro del otro. El depósito se encontró intacto, exento y oculto en un ni- cho debajo de una piedra de mediano tamaño, entre un caos de bloques caídos del techo. No se encuentra asociado a restos óseos o de otra naturaleza. Constatamos la presencia de numerosos fragmentos cerámicos, todos elaborados a mano, debajo y entre otras piedras de la sala, lo que indica que la colocación del depósito cerámico no debió ser un hecho puntual.

Dado que esta zona es transitada por visitantes del Parque Natural del Alto Tajo y espeleólogos se solicitó una intervención excepcional para preservar los restos hallados. Procedimos a su extracción documentada, así como a un levantamiento topográfico para situarlos espacialmente, siendo trasladados al Museo de Guadalajara. Para la elaboración de la topografía se utilizó un medidor Leica Dixto 310 con la placa modificada DixtoX2 para espeleología, utilizando el programa Topodroid.

El depósito consiste en un primer recipiente elaborado a mano, con una altura de $8,2 \mathrm{~cm}$ y un diámetro de $22,5 \mathrm{~cm}$ en forma de escudilla con carena alta y de factura grosera, que presenta restos de una posible asita en cinta o mamelón desaparecido en su borde. Contenía en su interior residuos de aspecto carbonoso, extraídos en el laboratorio del Museo de Guadalajara.

Esta escudilla contenía un recipiente globular en forma de ollita con la base umbilicada y el borde ligeramente exvasado, con una altura máxima de $12,7 \mathrm{~cm}$, diámetro máximo de 9,4 y diámetro en la boca de 7,8 $\mathrm{cm}$. Presenta decoración en los dos tercios superiores formada por líneas paralelas y triángulos conectados, o "dientes de lobo", trazados con una línea incisa fina, con un acabado regular, rellenos en los dos frisos con 

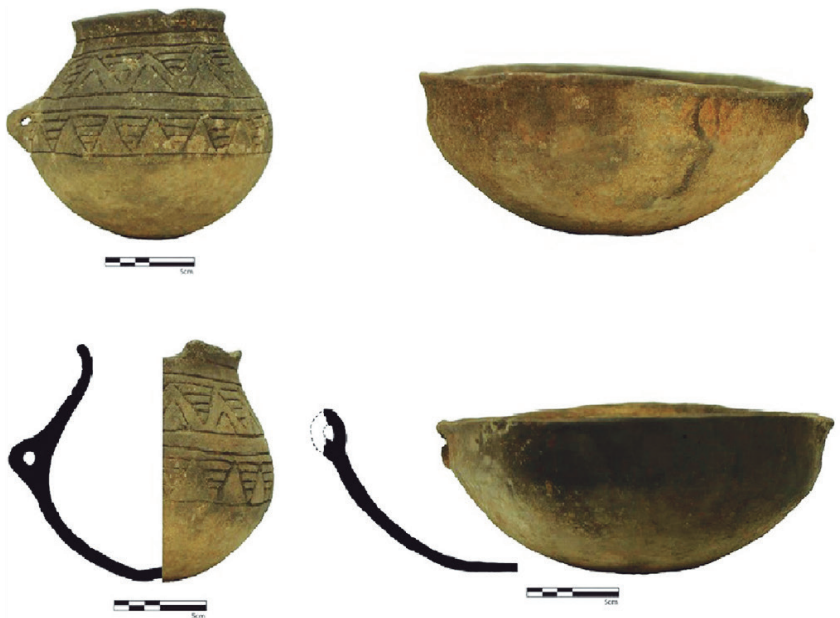

Fig. 5. Depósito cerámico. / Pottery deposit.

líneas paralelas. Los residuos de ambos recipientes se recogieron diferenciando los que estaban más próximos $\mathrm{o}$ adheridos a la pared de los vasos del resto. Esta muestra nos dio un total de $185 \mathrm{~g}$ de residuo para la escudilla y $18 \mathrm{~g}$ para el recipiente globular.

Separamos una muestra del contenido de este último para datación con C14. El depósito estaba cubierto por un clasto de mediano tamaño, pero no estaba sellado, por lo que no podemos asegurar la ausencia de contaminaciones.

El resultado obtenido de la muestra analizada en Beta Analytic calibrado a dos sigmas ofrece un resultado coherente con la tipología cerámica del contenedor:

\begin{tabular}{|c|c|c|c|}
\hline Laboratorio & $\begin{array}{c}\text { Tipo de } \\
\text { Muestra }\end{array}$ & $\begin{array}{c}\text { Fecha C14 } \\
\text { convencional }\end{array}$ & $\begin{array}{c}\text { Calibración } \\
\mathbf{2} \text { sigmas: }\end{array}$ \\
\hline Beta - $\mathbf{5 5 5 2 7 3}$ & $\begin{array}{c}\text { Material car- } \\
\text { bonizado }\end{array}$ & $2910 \pm 30$ B.P. & $\begin{array}{l}1211-1012 \\
\text { CAL. B.C. }\end{array}$ \\
\hline
\end{tabular}

Tabla 1: Datación del contenido del recipiente cerámico superior del depósito. / Dating of the deposit's upper vessel contents.

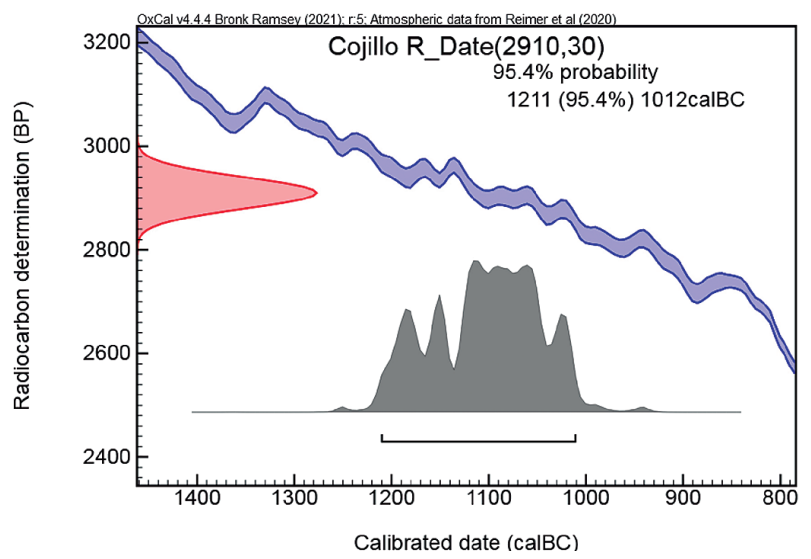

Fig. 6. Resultados de la datación calibrada de la muestra. / Results of calibrated dating of the sample. (OxCal v4.4.4 Bronk Ramsey (2021); r.5: Atmospheric data from Reimer et al (2020). IntCal20.

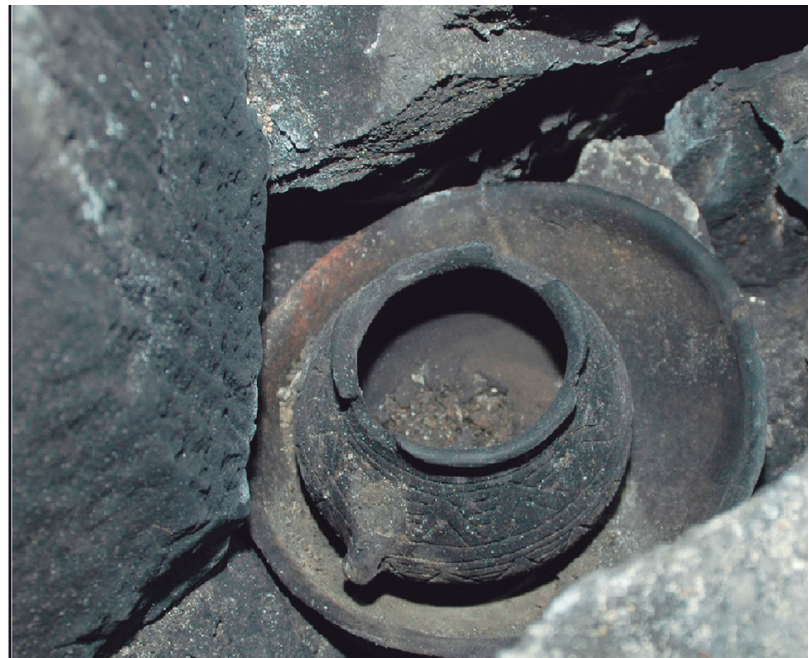

\subsection{Implicaciones cronoculturales}

A pesar de la coherencia de la fecha obtenida con la tipología de los vasos, carecemos de contexto cronoestratigráfico que pudiera clarificar el tipo de uso que se dio a la cueva, así como de las posibles secuencias culturales que pudiera ofrecer

Resaltamos las circunstancias en las que se halló el depósito. Es evidente el ánimo de ocultación del conjunto, así como su deliberada disposición y aparente carácter ritual, apareciendo en una zona de la cueva con pocas posibilidades habitacionales.

La presencia de elementos de molienda, cerámica y restos de fauna en el vestíbulo y corredor principal de la cueva pueden indicar un uso habitacional próximo la entrada, pero su hallazgo en superficie y la ausencia de elementos cerámicos diagnósticos aclara poco sobre su sincronía o no con el conjunto vascular encontrado.

En el área geográfica en la que se enmarca la cueva del Cojillo se han excavado y publicado dos yacimientos con ocupaciones durante el Bronce final: la necrópolis de Herrería, de la que hablaremos posteriormente, y el cercano yacimiento de Fuente Estaca, con fechas posteriores: 919 cal. B.C. (Martínez-Sastre, 1992). Hasta el 2007 se conocían pocas localizaciones publicadas para esta zona y el interfluvio Mesa, Piedra, Jalón (Martínez Naranjo, 1997; Arenas, 1999: 171-176), ambas resultado de prospecciones superficiales. Esta aparente ausencia de poblamiento durante el Bronce final podía ser achacable al vacío investigador (Barroso, 2002), enmendado en parte gracias a los trabajos de prospección superficiales realizados para las Cartas Arqueológicas entre 2007 y 2009 y "El Paleolítico en el Alto Tajo" en 2017. Se constata la ocupación generalizadas de cuevas y abrigos durante la Prehistoria Reciente, además de indicios de una ocupación del territorio durante el Bronce final más intensa y diversifi- 
cada, en su mayoría poblados más o menos extensos situados en vegas fértiles y áreas bajas de los valles, con presencia de vasos carenados y decoración incisa y acanalada en términos como Alustante, Alcoroches, Piqueras y Terzaga (vertiente del Tajo), y en Hinojosa, Tortuera, Embid, Mochales y Anchuela del Campo (vertiente del Ebro) (Arenas, 2011). Su estudio puede ayudar a contextualizar de forma más precisa el Bronce final local.

Los yacimientos datados con cronologías similares más próximos a la cueva del Cojillo los encontramos en: Pico Buitre, poblado situado en el valle alto del Henares (Espinosa de Henares, Guadalajara): 3080-2900 B.P. y 2990-2810 B.P. (Crespo, 1992; Castro et al., 1996); el poblado de El Macerado (Leciñena, Zaragoza): 29752925 B.P. y 2950-2870 B.P. (Rodanés y Picazo, 1997) o en la Sima del Ruidor (Aldehuela, Teruel): 3110-3010 B.P. (Picazo, 1986).

Hemos de apuntar la dificultad de extrapolar los estudios de otras áreas con otras condiciones geográficas y procesos culturales posiblemente diferentes (Cerdeño y Sagardoy, 2016: 26).

La ocupación de cuevas como lugares de habitación y/o como lugar de enterramiento durante el Bronce y el Bronce final es habitual, como se constata en el Portalón de Cueva Mayor (Burgos) (Pérez-Romero et al., 2016), en el valle del Ebro (Rodanés, 1997), así como en la limítrofe provincia de Teruel (Burillo y Picazo, 1994), o en el levante peninsular, en Castellón (Palomar, 1991). Tampoco es nueva la existencia de cuevas en las que conviven espacios diferenciados, habitacionales y sepulcrales, durante el Bronce final, como se comprueba en la cueva riojana de San Bartolomé (Rodanés, 1997).

Destacamos aquí los resultados obtenidos en la necrópolis de Herrería, en sus niveles I y II (Cerdeño y Sagardoy, 2016). Situada a unos $40 \mathrm{Km}$ del Cojillo, aunque fuera del contexto serrano, las autoras identifican para la fase I 74 tumbas de incineración, marcadas con estelas con escaso ajuar asociado, exclusivamente lítico, ofrendas de fauna y cantos rodados, fechada con C14 en el siglo XI a. C. (XIII cal. a. C.). En la fase II, datada con C14 en el siglo IX a. C. (X cal. a. C.), se han documentado 196 tumbas en las que se practica tanto la inhumación como la cremación, en túmulos señalados con o sin estela central. Aparecen cerámicas incisas, excisas y acanaladas, decoradas con cordones, así como ajuares en bronce, ámbar báltico e incluso un fragmento de oro. La datación del conjunto de la cueva del Cojillo nos situaría entre estas fases de ocupación de la necrópolis, entre el Bronce final II y el III, según la periodización propuesta por estas autoras.

Dataciones absolutas con cronologías similares las encontramos en otra necrópolis de incineración, en San Pedro (Oncala, al nordeste de Soria), (1270-1010 cal. B.C. y 1260-910 cal. B.C.) (Tabernero et al., 2010).

\section{EL DISPOSITIVO GRÁFICO \\ 4.1. Metodología de registro}

Para la documentación gráfica se ha utilizado una cámara réflex Pentax K1 Mark II y una Canon EOS 200D, con el empleo de iluminación artificial rasante para los grabados. Debido a la situación de los paneles y sus dimensiones se ha realizado una restitución fotogramétrica con dos objetivos: la obtención de una ortofotografía que permita la visualización del panel completo a alta calidad y un modelo tridimensional para la salvaguarda de las grafías. Ambos propósitos han sido llevados a cabo a través del software Agisoft Metashape $®$.

Los motivos han sido descritos según la clasificación de Apellániz y Uribarri para los tipos de Atapuerca (1976). Únicamente se han numerado las unidades gráficas asimilables como tales, algo que pasa por su aislamiento o similitud con formas clasificables. Se utilizan por tanto las denominaciones unidad gráfica y agrupación gráfica, en consonancia con las Unidades de Expresión descritas en los trabajos de Cueva Maja (Samaniego et al., 2001: 137).

\subsection{Descripción}

El dispositivo gráfico comienza a unos veinte metros de la entrada y se extiende casi ininterrumpidamente hasta su abrupto cese. El tramo decorado se ha dividido en tres conjuntos: el primero corresponde a la parte final del corredor de entrada y los dos siguientes a ensanchamientos o "salas" naturales delimitadas por obstáculos topográficos. El programa iconográfico se compone esencialmente de trazos grabados, rectilíneos y subparalelos situados de forma preferencial en el techo de las salas principales. La pintura se reserva para el primer conjunto, débilmente iluminado por la luz exterior.

El grado de alteración de algunos surcos impide descartar su origen físico o biológico, por ello en los calcos solo se muestran aquello que in situ presenta un carácter antrópico más evidente.

\subsubsection{Conjunto I}

Se compone de tres paneles situados a la misma distancia de la entrada, en la parte final de la primera galería. Ocupan la pared izquierda, el techo y la pared derecha, en pigmento rojo, grabado y pigmento negro, respectivamente. A partir de este punto, las grafías se ejecutan exclusivamente mediante grabado.

Panel 1: Dimensiones: $21 \times 184 \mathrm{~cm}$. Ocupa un estrecho friso natural situado en la pared izquierda. Cuenta con cinco unidades gráficas en pigmento rojo, homogéneas en color y dimensiones. La mejor conservada (Fig. 10: 2) parece ser una puntuación, interpretación que haremos extensible al resto de elementos del panel, si bien de dos de ellos solo quedan manchas de color muy desvaídas.

Panel 2: Dimensiones $207 \times 130 \mathrm{~cm}$. Se corresponde con una fracción del techo delimitada por diaclasas y 


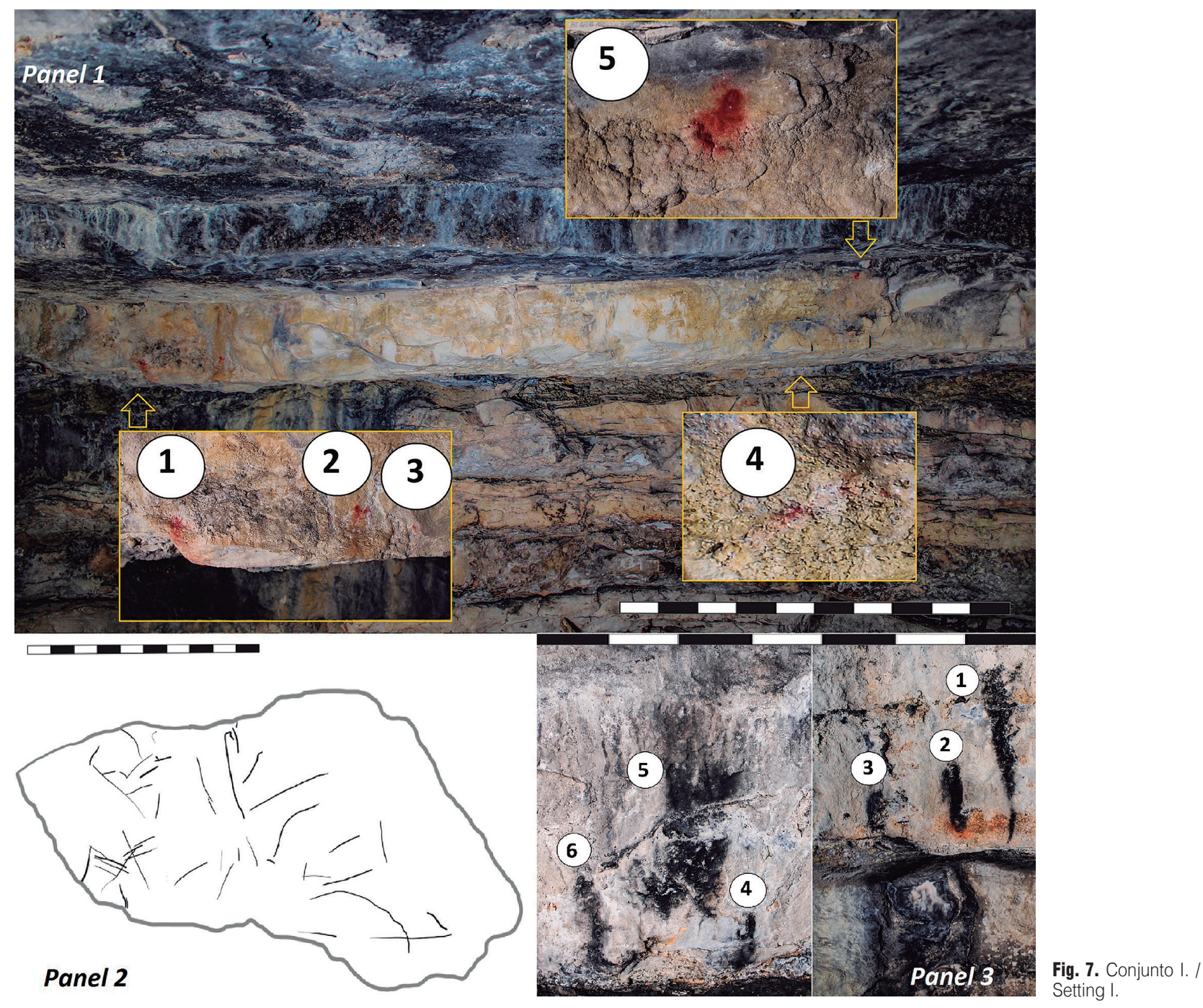

contiene una única agrupación gráfica. Lo componen líneas grabadas independientes de surco profundo en $\cup$, sin orientación preferencial.

Pane/ 3: Dimensiones: $35 \times 385 \mathrm{~cm}$. Se divide en dos sectores separados que contienen 6 unidades gráficas ejecutadas en negro. El primer sector, más cercano a la boca de la cavidad, se compone de tres trazos verticales. El segundo presenta una gran mancha central flanqueada por dos trazos menores. Es probable que la mancha central tenga su origen en algún sistema de iluminación, como sugiere la presencia de huellas de fuego en la repisa inferior.

\subsubsection{Conjunto II}

El segundo conjunto, dividido en tres paneles, aunque topográficamente continuo, se desarrolla a lo largo de los primeros cuatro metros del techo de esta segunda sala. La división responde a fracturas o coladas que interrumpen brevemente el desarrollo del dispositivo gráfico. Todo el conjunto se ha realizado mediante grabado con objeto apuntado o romo, indistintamente, en algunas zonas suavizado por alteraciones posteriores. La presencia de formas de aspecto geométrico es, a nuestro parecer, fortuita, aunque mencionamos algunos paralelos formales.

Panel 1: Dimensiones: $163 \times 84$. Muestra una gran acumulación de líneas en diferentes estados de alteración que, pese a ello, conservan el surco definido. Los trazos siguen principalmente dos direcciones que en algunos puntos se cruzan perpendicularmente. Se concentran en dos agrupaciones gráficas, dejando el centro más libre, posiblemente por su mayor grado de erosión. En la parte inferior del panel se podría apreciar la forma J6, pero la maraña de trazos impide asegurarlo. Destacamos dos posibles figuras, un escaleriforme (H5. Fig. 10: 3) y una forma cuadrangular (A3).

Panel 2: Dimensiones: $172 \times 120$. Contiene una escueta cantidad de líneas y en él se sugieren una posible forma ovalada o fusiforme. El resto computa como 


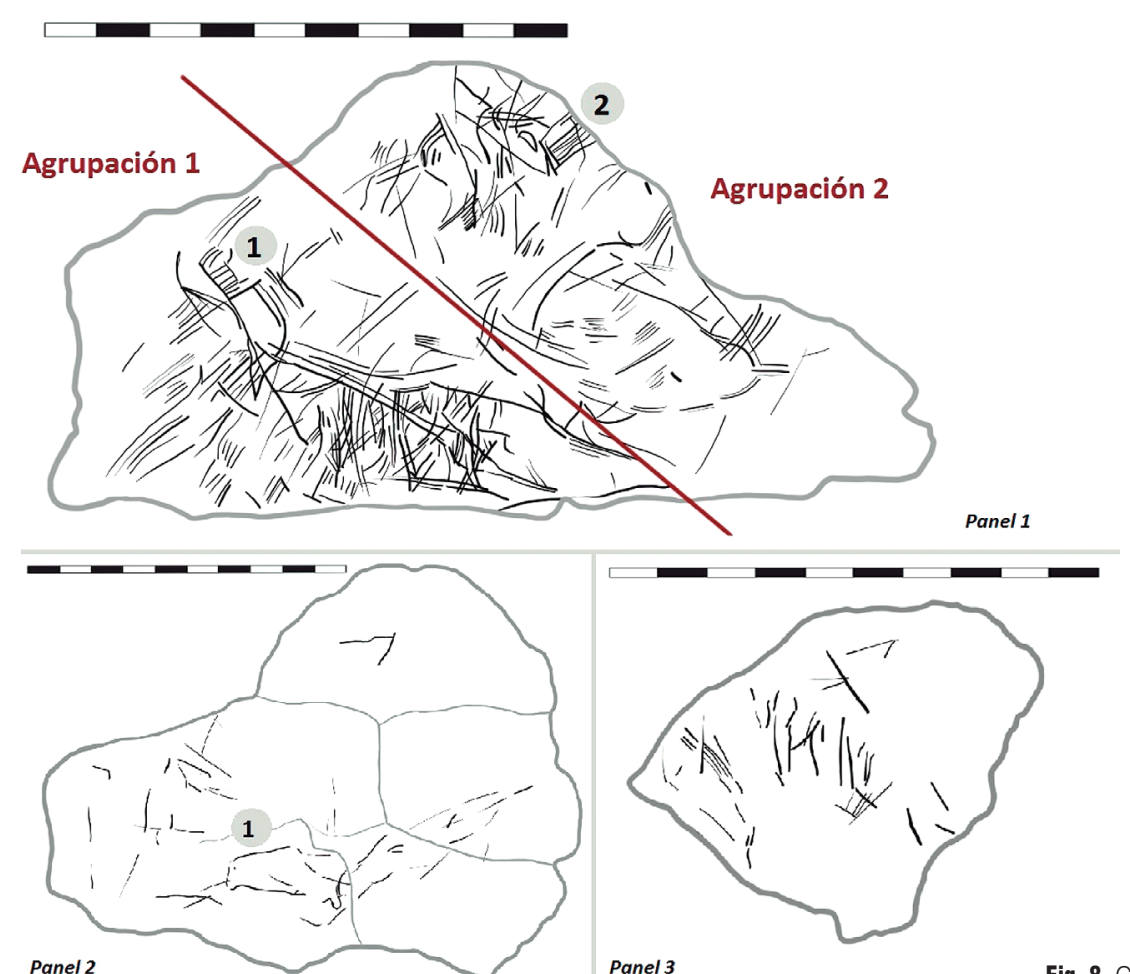

Panel 2

Panel 3

una única unidad gráfica. En la parte más avanzada del panel se observan algunos trazos infrapuestos a una colada estalagmítica. Este hecho valida la antigüedad de las líneas, de idéntico carácter a las del resto del panel.

Panel 3: Dimensiones: $90 \times 70 \mathrm{~cm}$. Consta de una única agrupación gráfica que engarza con el panel previo. Contiene trazos similares a las anteriores que se infraponen a los crecimientos calcíticos, en algunos casos con un notable desarrollo.

\subsubsection{Conjunto III}

El último conjunto decorado se compone de dos paneles situados sobre el depósito cerámico recuperado. Aquí el grado de alteración de la pared es menor y los grabados se conservan en buen estado (Fig. 10: 1). Se encuentran también en el techo de la cavidad, en
Fig. 8. Conjunto II. / Setting II. el interior de un panel horizontal delimitado por superficies más alteradas.

Panel 1: Dimensiones: $187 \times 117 \mathrm{~cm}$. Se aprecian tres agrupaciones gráficas de trazos subparalelos con algunas líneas que los cruzan. Cada agrupación podría contener formas $\mathrm{H} 5$ pero su aislamiento es casi inapreciable, por lo que no han sido consideradas unidades gráficas individuales. Las superposiciones, apreciables a simple vista, indican que los trazos subparalelos no han sido realizados de forma mecánica, puesto que las líneas que los cortan se superponen e infraponen de modo indistinto.

Panel 2: Dimensiones: $73 \times 64 \mathrm{~cm}$. El último panel decorado de la cueva se sitúa igualmente en el techo, a una altura considerable $(207 \mathrm{~cm})$, y en él se aprecian unos pocos trazos subparalelos que conforman una única unidad gráfica. Uno de ellos tiene aspecto serpentiforme (K5), forma no habitual en el resto de paneles.
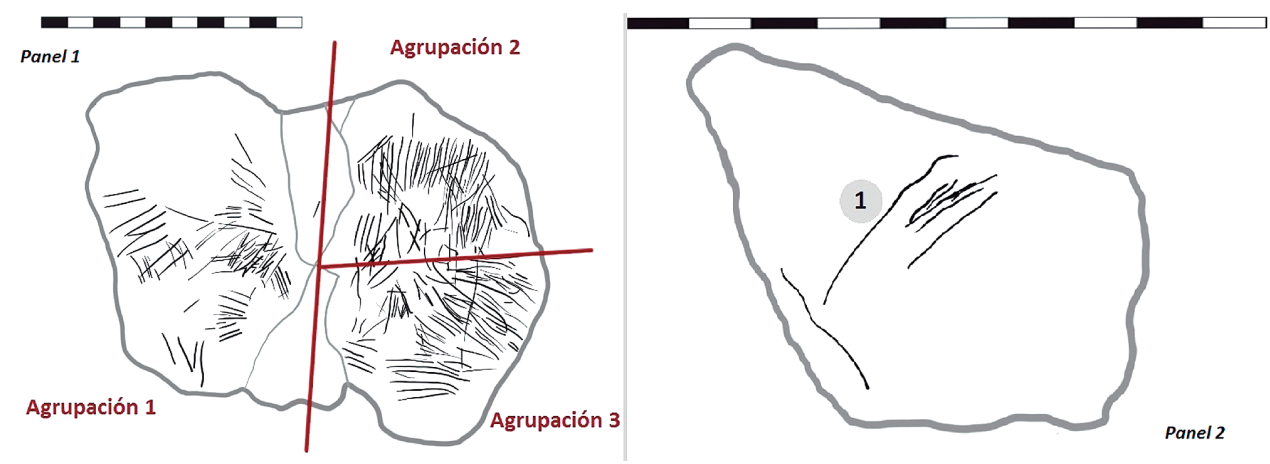

Fig. 9. Conjunto III. / Setting III. 


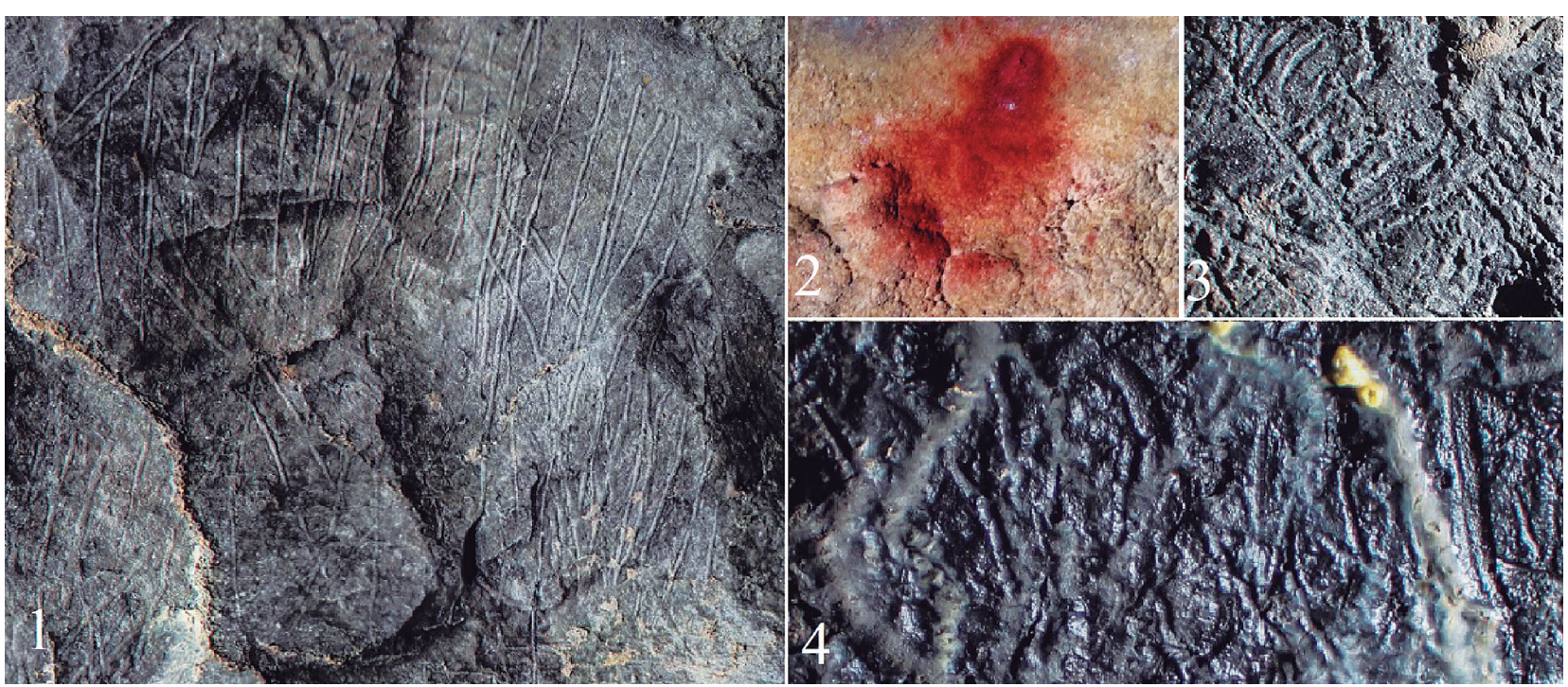

Fig. 10. Detalles de las grafías: 1. Conj. III Pan. 1; 2. Conj. I Pan. 1; 3. Conj. II Pan. 1; 4. Conj. II Pan. 3. / Paintings and engravings details: 1. Set. III Pan. 1; 2. Set. I Pan. 1; 3. Set. II Pan. 1; 4. Set. I| Pan. 3.

\subsection{Valoración}

El Arte Rupestre Pospaleolítico de la provincia de Guadalajara se encuentra desigualmente repartido. Solo su sector occidental cuenta con un núcleo de cierta entidad, compartido con el este madrileño (Alcolea-González, 1993). Dentro del Arte Esquemático típico se encuentran el Covacho del Ocejón (Sebastián y Gómez-Barrera, 2003), Portalón de Villacadima (Ortego, 1963), abrigo de los Forestales (Oliver et al., 2015), abrigos del Llano y Rillo II (Balbín et al., 1989) y las cuevas del Arroyo de la Vega, Los Hombres (Alcolea-González et al., 1993) y Las Ovejas (Balbín, 2002). Para el grabado, el yacimiento de Rillo de Gallo (Alcolea-González et al., 1994), Peña Escrita (Cerdeño y García-Huerta, 1983) y cueva del Robusto (Arenas, 2010). Existen noticias de grabados en Sigüenza (Morere, 1983), Atienza (Puche y Sierra, 2012) y en el norte de la provincia (Cabré, 1941), con escasa o nula documentación. En el marco de una reciente tesis doctoral, se dio noticia de nuevos yacimientos en el área de Molina de Aragón, entre los que destacan los abrigos de Peñahíta, Los Casares III y El Hocino, con Arte Esquemático y Levantino (Lancharro 2015). Damos por perdidas las grafías desaparecidas bajo el embalse de Beleña, en el barranco de las Quintillas (Ortego, 1979).

En el interior peninsular, con especial arraigo en la submeseta norte, existe una variedad de Arte Pospaleolítico ligado al ámbito subterráneo. Este horizonte se compone de esquemas ejecutados mediante grabado, con una fuerte abstracción, que dificulta en la mayoría de las ocasiones la identificación de figura alguna. Dependiendo de su situación geográfica, ha recibido las denominaciones de Grupo Solacueva-Galería del Sílex (Apellániz y Uribarri, 1976) o Sector Sudoriental de la Cuenca del Duero (Municio y Zamora, 1989). Podemos incluir en esta tipología las cuevas de los Enebralejos, la Vaquera y la Griega en Segovia (Municio y Zamora, 1989), San Bartolomé de Ucero, cueva del Asno, Covarrubias de Ciria (Gómez-Barrera, 1992; Samaniego, 1999) y Cueva Maja (Samaniego et al., 2001) en Soria y, con reservas, el Reguerillo en Madrid (Mas et al., 2010). En Atapuerca, en diferentes emplazamientos que incluyen Galería del Sílex (Apellániz y Uribarri, 1976), Salón del Coro, Cueva del Silo, Galería del Silo, Galería Baja y Galería de las Estatuas (García-Díez et al., 2001; 2003). Para la provincia de Guadalajara, así como para la Submeseta sur en su conjunto, el caso que aquí se describe supone el primer hallazgo conocido.

Algunas cavidades que conjugan grafías y depósito han servido para acotar una cronología que parte desde los inicios de la Prehistoria reciente, con una mayor concentración en el Bronce antiguo. El caso más paradigmático lo ilustra la cueva de los Enebralejos, en Segovia, considerada un conjunto cerrado. Contiene abundantes inhumaciones individuales asociadas a grafías adscritas al Calcolítico y el Bronce antiguo (Municio y Zamora, 1989). En el complejo kárstico de Atapuerca, aparecen motivos similares a lo largo de la Galería del Sílex (Apellániz, 1976) con dataciones directas sobre pigmento de 2142-1603 B.C. y 2143-1937 B.C (García-Díez et al., 2003). Otras tres fechas directas para la Sala de la Fuente, en Ojo Guareña: 4416 a 4146 cal. B.P.; 4448 a 4426 cal. B.P. y 5747 a 5583 cal. B. P. (Gómez-Barrera et al., 2000). Las dataciones efectuadas sobre los depósitos arqueológicos de las cuevas sorianas de La Maja y el Asno I y II (Samaniego et al., 2001: 115) ofrecen cronologías similares.

Este horizonte gráfico parece cesar abruptamente al sur del Sistema central. En su vertiente meridional, el atestiguado fenómeno rupestre y funerario en cueva (Alcolea-González et al. 1993) se limita al ámbito de la pintura, con una cronología neolítico-calcolítica. La presen- 
cia de paralelos para este arte esquemático "típico" en el segoviano núcleo del Duratón, invalida una dualidad pintura/grabado como hipótesis para los límites geográficos de las manifestaciones objeto de este artículo.

En el occidente de Guadalajara, la existencia de cavidades con evidencias del Bronce medio-final de forma exclusiva, carentes de grafías (Ortego 1979; Ruiz Pérez 1991; Jiménez y Barroso 2000), circunstancia extensible a yacimientos de Toledo (Montero et al. 1999) o Cuenca (Díaz-Andreu 1990), apuntan a una clara interrupción gráfica. Una posible excepción es la cueva de Pedro-Fernández Villacañas (Madrid) (Sánchez Meseguer 2006), que contiene restos humanos y un escueto dispositivo gráfico que no permite sacar conclusiones sobre su intencionalidad. Asimismo, en el sur de la provincia de Ciudad Real, lindando con Jaén, encontramos enterramiento y grafías en una cavidad natural monumentalizada de Castillejo de Bonete (Polo Martin et al. 2015). Conjuga grabado y pintura $y$, pese a la separación geográfica, permite apuntar similitudes de ordenamiento espacial que se tratarán en la discusión.

\section{DISCUSIÓN}

La dimensión geográfica de los dispositivos parietales análogos a la cueva del Cojillo no ha sido claramente definida. Esto queda ejemplificado en las diferentes denominaciones existentes; de entre estas, hemos escogido Arte Pospaleolítico en cueva como el más aséptico. Aunque es difícil proponer unos límites para un comportamiento simbólico cuya principal característica es la ausencia de figuraciones bien definidas, el registro apunta al alto valle del Duero como área en torno a la cual se articula el fenómeno. Las estribaciones de la cordillera Cantábrica al norte y el sistema Central al sur enmarcan el área cuyos límites se hacen más difusos a este y oeste. El Cojillo supone una trasgresión a través del sistema lbérico, quizá condicionada por factores litológicos y por las posibilidades de comunicación Duero-Tajo a través de los Altos de Barahona. Desde una perspectiva geográfico-cultural, el Cojillo se sitúa en el punto de convergencia entre el área de contacto de los momentos tardíos de Cogotas I, la desintegración del Bronce manchego y la llegada de los Campos de Urnas. El área de distribución de los yacimientos análogos ocupa gran parte del horizonte Cogotas I, sin embargo, los riesgos de correlacionar áreas culturales y registro material ya han sido expuestos en varias ocasiones (Blanco González 2011).

Más difícil es intentar aproximarse a la ordenación interna del yacimiento. El primer rasgo diferenciador es que, a excepción de la primera sala, las grafías son exclusivamente cenitales, pese a la existencia de paredes perfectamente adecuadas para su decoración. Este hecho no se comparte con ningún otro yacimiento del grupo. El segundo rasgo es la existencia de un único conjunto de grabados en cada sala profunda que, pese a haber sido interrumpidos por obstáculos, forman un continuum que no admite grafías externas.

Si atendemos a los paralelos más cercanos, en las cuevas de Enebralejos y La Vaquera, las grafías guardan una relación estrecha con los enterramientos; son "elementos definidores de espacios concretos" (Municio y Zamora 1989: 274), una relación que en el Cojillo solo podemos hacer coincidir con el depósito.

Más reveladora es la disposición de Cueva Maja (Samaniego et al. 2001). Pese a que la variedad morfológica de sus grabados es mayor respecto al Cojillo, se pueden establecer algunos paralelismos. Los grupos O-P de Cueva Maja, situados en la Cámara, guardan una estrecha similitud formal con los conjuntos II y II del Cojillo. Además, al fondo del Camarín grande, final del espacio decorado encontramos una serie de trazos (Grupo I), análogos a nuestro último panel. Comparten en ambas cuevas espacios interiores y apartados, lejos de las posibilidades habitacionales de la entrada. En la Cámara de Cueva Maja los materiales incluyen cerámica con motivos análogos a los grabados. Es plausible establecer un acondicionamiento similar en el Cojillo, tras un primer pasillo alcanzado por la luz diurna, se produce una sucesión de salas con implicaciones simbólicas.

Por último, en la cueva del Castillejo del Bonete (Polo Martín et al. 2015), en el extremo meridional de la Submeseta sur, se repite la asociación indicada para Enebralejos: acumulación gráfica en espacios funerarios. Salvando las distancias temáticas y geográficas, se puede plantear la coexistencia de expresiones locales propias unidas por un esquema común interregional.

\section{CONCLUSIONES}

No podemos conocer, dado el carácter prospectivo de los trabajos realizados, la intencionalidad de los autores de la ocultación del depósito y de las grafías, ni su posible funcionalidad. Dadas sus características podría estar relacionado con un comportamiento simbólico compartido, reproducido en un área cultural que ocupa gran parte de la Submeseta norte, ocasionalmente presente en la Submeseta sur. Cabría plantearse (Palomar, 1991; Rodanés, 1997; Abarquero et al., 2005) el carácter funerario del conjunto vascular, una hipótesis con evidencia insuficiente por ahora. De ser así, podríamos encontrarnos ante la convivencia de dos tipos de prácticas funerarias diferentes durante el Bronce final en un mismo territorio; con la irrupción del rito incinerador y el cambio de localización de las necrópolis hacia los espacios abiertos, que conlleva el progresivo abandono del hábitat en cuevas y su uso como espacio sepulcral. Se trata, en cualquier caso, de la primera evidencia de la utilización de cuevas durante el Bronce final en este territorio, lo que amplía el elenco de actividades y ambientes físicos frecuentados. La problemática adicional consiste en encajar una datación del Bronce final con unas grafías que indican, según los paralelos propuestos, cronologías más antiguas. La 
explicación más plausible conduce a una ejecución de las grafías sincrónica al depósito, con unas raíces culturales que tienen su origen en momentos más antiguos del Bronce local.

\section{AGRADECIMIENTOS}

Agradecemos al Centro de Estudios de Molina y Alto Tajo, por la financiación para la datación, a Pedro Guillén, Víctor Marfil y a José Ángel Jambrina, que detectó el depósito cerámico, por el apoyo prestado en la exploración de la cueva. A la Fundación Banco Sabadell por el apoyo económico a la investigación predoctoral de uno de los firmantes. A Teresa Sagardoy y José-Manuel Maíllo, por sus comentarios y apoyo logístico. Al equipo del proyecto de investigación Multipaleoiberia (ERC-2018-STG-805478), por su asesoramiento y la aportación del equipamiento necesario para el registro gráfico.

\section{BIBLIOGRAFÍA}

Abarquero, F. J., Palomino, A. L., Negredo, M. J., 2005. La cueva de La Revilla, un enterramiento colectivo del Bronce protocogotas en la sierra de Atapuerca (Burgos). BSAA Arqueología: Boletín del Seminario de Estudios de Arqueología 71, 89-136.

Alcaraz-Castaño, M., Weniger, G. C., Alcolea-González, J., De Andrés-Herrero, M., Baena, J., Balbín, R., Bolin, V., ... e Yravedra, J., 2015. Regreso a la Cueva de los Casares (Guadalajara). Un nuevo proyecto de investigación para el yacimiento del Seno A. Arqueología y Prehistoria del Interior peninsular 2, Área de Prehistoria de la Universidad de Alcalá de Henares, 68-89.

Alcolea-González. J., Bunes, F., García-Valero, M. A., Jiménez, P. J., 1993. Arte esquemático en la vertiente sur del Sistema Central. Congreso de Arqueología Peninsular, 199-216.

Alcolea-González, J., Jiménez, P., Barroso, R. M., 1994. Los grabados rupestres esquemáticos al aire libre de Rillo de Gallo (Guadalajara). Actas del congreso de Arqueología de la Edad del Bronce en Castilla-La Mancha, Toledo.

Apellániz, J.M., Uribarri, J.L., 1976. Estudios sobre Atapuerca (Burgos). 1. El Santuario de la Galería del Sílex. Cuadernos de Arqueología de Deusto 5. Bilbao.

Apellániz, J. M., 1982. El arte prehistórico del País Vasco y sus vecinos. Desclée de Brouwer, Bilbao.

Arenas, J. A. 1999. La Edad del Hierro en el Sistema Ibérico central, España. British Archaeological Reports, International Series, 780.

Arenas, J. A., 2010. Sobre la identificación de entornos religiosos en el horizonte prerromano celtibérico. En: Burillo, F. (Ed.), Estudios Celtibéricos, 6. VI Simposio sobre Celtíberos: Ritos y Mitos, 87-102. Fundación Segeda. Centro de Estudios Celtibéricos.

Arenas, J. A., 2011. El poblamiento prerromano en el área del Alto Tajo-Alto Jalón/The pre-Roman settlement in the Upper Tagus-Upper Jalón basins. Complutum 22(2), 129-146.

Balbin, R., Bueno, P., Jiménez, P., Alcolea-González, J., Fernández, J. A, Pino, E., Redondo, J.C., 1989. El abrigo rupestre del Llano, Rillo de Gallo, Molina de Aragón. XIX Congreso Nacional de Arqueología. 179-194. Zaragoza.
Balbín R., Bueno P., Jiménez P., Alcolea-González J., Fernández J.A., Pino E., Redondo, J.C., 1989. El yacimiento de Rillo de Gallo (Guadalajara). Wad-al-Hayara 16, 31-73.

Balbín R., 2001. Estado actual de la investigación del Arte Paleolítico en Guadalajara. En: Actas del primer Simposio de Arqueología de Guadalajara: Sigüenza, 4-7 octubre de 2000, 187-228. Ayuntamiento de Sigüenza.

Barandiarán, I., Altuna, J., 1973. La Cueva de los Casares (en Riba de Saelices, Guadalajara). Ministerio de educación y Ciencia, Dirección General de Bellas Artes, Comisaría General de Excavaciones Arqueológicas.

Barroso, R., 2002. El Bronce Final y los comienzos de la Edad del Hierro en el Tajo Superior. Servicio Publicaciones de la Universidad de Alcalá de Henares.

Blanco González, A. 2011. Práctica social, memoria y ritual en Cogotas I: esbozo teórico para un enfoque renovado. Trabajos de Prehistoria 68(1), 123-146.

Breuil, H., 1933-1935. Les peintures rupestre d'Espagne. Lagny.

Burillo, F., Picazo, J. V., 1994. El Bronce Medio y la transición al Bronce Tardío en Teruel. Gala 3-5.

Cabré, J., 1941. Pinturas y grabados rupestres, esquemáticos, de las provincias de Soria y Segovia. Archivo Español de Arqueología XLIII, 316-344.

Castro Martínez, P. V., Lull, V., Mico, L. R., 1996. Cronología de la Prehistoria Reciente de la Península Ibérica y Baleares (c. 2800-900 cal ANE). British Archaeological Reports, International Series, 652.

Cerdeño, M. L., García Huerta, R., 1983. Noticia preliminar de los grabados de la Peña Escrita (Canales de Molina, Guadalajara). Zephyrus 36, 179-186.

Cerdeño, M. L., Sagardoy, T., 2016. La necrópolis de Herrería I y |I. Las fases culturales del Bronce Final II-III. La Ergástula, Madrid.

Cerralbo, M. D., 1911. Páginas de la historia patria, por mis excavaciones arqueológicas. Inédito.

Crespo, M. L., 1992. Pico Buitre y el Bronce Final en el valle del Henares. En: Valiente Malla, J., La Celtización del Tajo Superior. Memorias del Seminario de Historia Antigua III. Alcalá de Henares, 45-65.

Díaz Andreu, M., 1990. La desigualdad social durante la edad del bronce en el sector septentrional de La Mancha: La Cueva de El Fraile (Saelices, Cuenca). Archivo de prehistoria levantina 20, 363-378.

Fernández-Tabera, M., Martín-Yebra, P., 1982. Catálogo de cavidades de Guadalajara. Federación Castellana Centro de Espeleología, Comisión de Catalogación de Cavidades. Madrid.

García-Díez, M., Martín, J., Ortega, A. I., Martín, M. A., 2001. Grafismo rupestre postpaleolítico en la Sierra de Atapuerca (Burgos): Salón del Coro, Galería del Silo, Galería Baja, Galería de las Estatuas y Cueva del Silo. Espacio, Tiempo y Forma Serie I, 227-259.

García Díez, M., Martín, J., Martín, M.A., Ortega, A.I., 2003. Dates for rock art at a Bronze Age sanctuary at the Galería del Sílex cave. Antiquity 77, 296.

Gómez-Barrera, J. A., 1992. Manifestaciones de la facies esquemática en el centro y norte de la Península Ibérica. Espacio, Tiempo y Forma 5, 231-264. 
Gómez-Barrera, J. A, Ortega, A. I., Martín, M. A., García, M., Fernández, J. J., Delval, J., 2000. Las manifestaciones gráficas de la Sala de la Fuente (Ojo Guareña, Burgos): dataciones absolutas para la contextualización del arte rupestre. Boletín del Seminario de Arte y Arqueología LXVI, 65-79.

Jiménez P. J., Barroso, R. M., 2000. Diversificación del hábitat del Bronce Medio en Guadalajara: la Cueva de La Vaca. Actas do $3^{\circ}$ Congresso de Arqueologia Peninsular V, 131-144.

Lancharro, M. A., 2015. Marcadores gráficos y territorios megalíticos en la cuenca interior del Tajo: Toledo, Madrid y Guadalajara. Tesis doctoral inédita. Universidad de Alcalá.

Llanos, A., 1966. Resumen tipológico del arte esquemático en el País Vasco. Estudios de Arqueología Alavesa 1, 149-158.

Martínez-Naranjo, J.P., 1997. El inicio del mundo celtibérico en el interfluvio del Alto Jalón-Mesa. Complutum 8, 161-182

Martínez-Sastre, V., 1992. El poblado de Campos de Urnas de Fuente Estaca (Embid, Guadalajara). La celtización del Tajo Superior, 67-78.

Mas, M., Torra, G., Maura, R., Solís, M., 2010. El arte parietal en la Cueva del Reguerillo. Patones (Madrid). Zephyrus 66, 79-92.

Montero, I., Rodríguez, S., Ruiz Taboada, A., 1999. La cueva-mina de La Serrana (Urda, Toledo) y su contribución al estudio del mundo funerario durante la Edad del Bronce en La Mancha. Anales Toledanos 37, 29-38.

Morère, N., 1983. Carta Arqueológica de la región seguntina. Institución Provincial de Cultura Marqués de Santillana. Guadalajara.

Municio, L., Zamora, A., 1989. Notas sobre grabados y pinturas asociados a necrópolis colectivas calcolíticas: los conjuntos de las cuevas de Los Enebralejos y de La Vaquera (Segovia). Trabajos de Prehistoria 46, 271-278.

Oliver, D., Sagardoy, T., Moreno, D., Bravo, F., 2015. Arte rupestre postpaleolítico en la provincia de Guadalajara: El Abrigo de los Forestales. Serie arqueológica 24, 565.607.

Ortego, T., 1963. Las pinturas rupestres de El Portalón, en el término de Villacadima (Guadalajara). Ampúrias 25, 61-104.

Ortego, T., 1979. Un nuevo abrigo con pinturas rupestres en el término de Muriel (Guadalajara). Actas del XV Congreso Nacional de Arqueología (Lugo, 1977), 429-437.

Palomar, V., 1991. Cuevas de enterramiento del Bronce Valenciano en el Alto Palancia (Castellón). Cuadernos de prehistoria y arqueología castellonenses 15, 93-114.

Peiro, R., Martín, F., 1990. El karst de la Muela de Utiel y Hoz Seca (Peralejos de las Truchas-Checa, Guadalajara). Garma 1.

Pérez-Romero, A., Alday, A., Iriarte, E., Francés-Negro, M., Galindo-Pellicena, M. A., Álvarez-Fernández, A., Juez, L., Arsuaga, J.L., Carretero, J. M., 2016. La cerámica de la Edad del Bronce en el yacimiento de El Portalón de Cueva Mayor (Sierra de Atapuerca, Burgos, España). Munibe Antropologia-Arkeologia 67, 105-126.

Picazo, J. V., 1991. Excavaciones arqueológicas en La Sima del Ruidor (Aldehuela, Teruel). Campaña de 1986. Arqueología Aragonesa 1986-1987, 119-125.

Polo, E., Bueno, P., Balbín, R. De, Benítez de Lugo, L., Palomares, N., 2015. Manifestaciones gráficas en la Cueva-Sima del Castillejo del Bonete (Terrinches, Ciudad Real). Arqueología del Interior Peninsular (Arpi) 2, 90-107.
Puche, I., Sierra, C., 2012. Descubrimiento de petroglifos en Atienza. Atienza de los Juglares 44. Atienza, 6-12.

Rodanés J. M., 1997. Las cuevas sepulcrales en La Rioja. Estudio histórico-arqueológico. Munibe Antropologia-Arkeologia 49, 77-93.

Rodanés, J. M., Picazo, J. V., 1997. Bronce final y primera edad del hierro en Aragón. Caesaraugusta 72, 155-215.

Ruiz Pérez, M., 1991. Materiales cerámicos de la cueva de la Galiana (Horche, Guadalajara). Wad-al-Hayara: Revista de estudios de Guadalajara 18, 377-408.

Samaniego, B., 1999. Espacios simbólicos en el Bronce Antiguo del Alto Duero. Complutum 14, 47-69.

Samaniego, B., Jimeno, A., Fernández-Moreno, J. J., Gómez-Barrera, J. A., 2001. Cueva Maja (Cabrejas del Pinar. Soria): Espacio y simbolismo en los inicios de la Edad del Bronce. Memorias Arqueología en Castilla y León 10. Junta de Castilla y León. Valladolid.

Sánchez Meseguer, J. L., 2006. Los grabados de la cueva de Pedro Fernández Villacañas (Estremera, Madrid). En: Lucas Pellicer, M. R., Cardito Rollán, L. M., Gómez Hernánz, J. (eds.), Dibujos en la roca. El arte rupestre en la Comunidad de Madrid. Madrid: Dirección General de Patrimonio Histórico de la Consejería de Cultura y Deportes de la Comunidad de Madrid, 345-358.

Sebastián, A., Gómez-Barrera, J. A., 2003. Las pinturas rupestres esquemáticas del Covacho del Ocejón (Valverde de los Arroyos, Guadalajara). Saldvie 3, 1-13.

Tabernero, C., Sanz, A., Benito, J. P., 2010. Necrópolis de cremación en el Nordeste de Soria. En: Burillo, F. (ed), VI Simposio sobre los Celtíberos. Mitos y Ritos. Estudios Celtibéricos, 6. Zaragoza, 391-402. 